\title{
Peristaltic flow of nanofluid in a deformable channel with double diffusion
}

\author{
Aamir Ali ${ }^{1}$. Y. Ali ${ }^{1}$ - D. N. Khan Marwat ${ }^{2}$ - M. Awais ${ }^{1} \cdot$ Z. Shah $^{3,4}$
}

Received: 28 October 2019 / Accepted: 7 December 2019 / Published online: 17 December 2019

(c) Springer Nature Switzerland AG 2019

\begin{abstract}
Fluid dynamics combined with double diffusive phenomenon in a deformable channel of peristaltically moving walls is investigated in this paper. The variable gap between heated walls is filled with nanofluids. The purpose is to examine the combined effects of surface deformation and peristaltic movement of the walls on the nanofluid flow along with coupled double diffusion analysis in a channel. We emphasized on the behavior of peristaltic flow with heat/mass transfer for nanofluid in the deformable channel whose walls are permeable and contracting or expanding in the normal direction. Nanofluids are widely utilized in industrial processes to boost the thermal diffusivity and conductivity. We have analyzed the effects of different involved parameters such as Reynolds number, surface deformation parameter, Prandtl number, wave number, Brownian and thermophoretic diffusion parameters on the flow fields, pressure distribution and concentration with the help of graphs. The results are shown graphically and discussed physically. It is noted that deformation increases the axial velocity and temperature of the fluid. For special cases, the current simulation and its solution are exactly matched with the classical models of viscous flow in a deformable channel of peristaltically moving walls.
\end{abstract}

Keywords Peristalsis · Double diffusion · Deformable channel · Nanofluid

\section{List of symbols}

$u, v \quad$ Axial and normal components of velocity

$T, C \quad$ Temperature and concentration of the fluid

$T_{1}, C_{1} \quad$ Upper wall temperature and concentration

$T_{0}, C_{0} \quad$ Lower wall temperature and concentration

$x, y \quad$ Cartesian coordinates

$t \quad$ Time

$v_{w} \quad$ Suction/injection velocity

$2 a(t) \quad$ Channel width

$D_{B}, D_{T} \quad$ Brownian diffusion and thermophoresis diffusion coefficients

$p \quad$ Pressure

$\lambda \quad$ Wavelength $b \quad$ Amplitude

$A \quad$ Suction/injection coefficient

$k, c_{p} \quad$ Thermal conductivity and specific heat

$\mathrm{Pr}$, Le Prandtl and Lewis numbers

$\theta, \phi \quad$ Non-dimensional temperature and concentration

Wall expansion ratio

Wave number

Reynolds number

Density

Non-dimensional $y$-coordinate

Kinematic viscosity

Aamir Ali, aamir_ali@cuiatk.edu.pk | ${ }^{1}$ Department of Mathematics, COMSATS University Islamabad, Attock Campus, Kamra Road, Attock 43600, Pakistan. ${ }^{2}$ Department of Mathematics, Faculty of Physical and Numerical Sciences, Islamia College Peshawar, Jamrod Road, University Campus, Peshawar, Khyber Pakhtunkhwa 25120, Pakistan. ${ }^{3}$ Center of Excellence in Theoretical and Computational Science (TaCS-CoE), SCL 802 Fixed Point Laboratory, Science Laboratory Building, King Mongkut's University of Technology Thonburi (KMUTT), 126 Pracha-Uthit Road, Bang Mod, Thrung Khru, Bangkok 10140, Thailand. ${ }^{4}$ KMUTT-Fixed Point Research Laboratory, Room SCL 802 Fixed Point Laboratory, Science Laboratory Building, Department of Mathematics, Faculty of Science, King Mongkut's University of Technology Thonburi (KMUTT), 126 Pracha-Uthit Road, Bang Mod, Thrung Khru, Bangkok 10140, Thailand. 
$\mu \quad$ Fluid viscosity

c Wave speed

\section{Introduction}

Channel flows are of particular importance and widely used in vibrating environments such as drilling oil wells, surgical operations during blood flow in the production, generation and preparing of food and paper, oil investigation and paper manufacturing. In science, it has applications for displaying respiratory functions in the lungs, modeling biochemical/clinical laboratories for analyzing chemicals/blood, etc. Channel flow with permeable walls was considered by Berman [1]. The properties of a fluid flow mechanism have been analyzed for 2D steady flow in rectangular cross sectional channel with porous walls. The Navies Stokes equations are simplified and solved analytically. He found the analytic solution subject to the noslip and symmetric boundary conditions. Due to increasing interest in many applications, number of studies are undertaken to address various situations of small and large suction/injection velocities [2-6].

Deformable channel is a geometry with expanding/ contracting porous walls. The flow of viscous fluid inside a semi-infinite, impermeable and contracting/growing pipe was early studied by Uchida and Aoki [7], in which the momentum equations were transformed by using similarity analysis both in space and time. In afterward investigation by Goto and Uchida [8], the similarity was recurring for a constricting tube with porous walls. Similarly flow among contracting or increasing parallel permeable walls was considered by Dauenhauer and Majdalani $[9,10]$. They studied the two-dimensional unsteady, laminar and incompressible flow between parallel plates, which are expanding or contracting. They treated full Navier-Stokes equations and do not consider the boundary layer approximations. They have taken two types of porosities at the porous surfaces of the channel, one is because of the expansion or contraction of the plates and the alternative is the common porosity. A strong mathematical relation is established between the two porosities in such a way they introduced an advanced parameter, which is called injection coefficient. It quantifies porosity of the plates and works as a control parameter in the ultimate solution. The study is further extended to applied problems in biomechanics with the assumptions of small cross flow Reynolds number (Re) and surfaces deformation rate $(\alpha)$. Berman is based on one physical parameter which is cross flow Reynolds number, while Majdalani et al. [11] are dealing with two limitations the Reynolds number and the plate expansion rate. They presented variables which reduces the NS equations into a nonlinear ODEs and solved the problem numerically. Later on, they give analytic solution of problem by using double perturbation procedure and the solutions are valid for small effects of both suction/injection parameter and wall expansion ratio. Most recent investigations of the channel flow problems with deformable walls are presented in [12-21]. The heat transfer phenomenon in a deformable porous channel was also discussed by Asghar et al. [22]. They provided homotopy solutions to the problem and compared the analytic results with the numerical solutions computed by RK method coupled with shooting technique.

Peristalsis is the train of sinusoidal waves resulting from contraction and expansion of an extensible channel propagate along the length of the channel. The mechanism is accountable for fluid transport in many physiological systems such as ureter push urine from kidney into bladder, contraction and expansion of muscle that occur throughout the digestive system. Peristaltic mechanism is fairly popular amongst the modern researchers due of its applications in engineering and physiological problems e.g. chyme wave in gastrointestinal tract, worm's locomotion, sanitary fluid transport, swallowing of food through esophagus and many others. Latham [23] has initiated the pioneering work on peristaltic transport for the first time in his MS Thesis. Several investigations of practical importance are presented since the pioneering work of Latham [23]. Some of these studies are given in [24-34]. Khan Marwat and Asghar [35] studied the peristaltic flow in deformable channel. They combined the peristaltic transport phenomenon with deformation in channels and found the perturbation solutions of the transformed problem.

The word nanofluid was introduced for the first time by Choi [36] and presented at occasion of ASME winter yearly conference. It is new class of heat transfer in fluids which contains nano-particles and fluid. To improve the performance of heat transfer in base fluids, it is experimented to include additives in the fluids. Nanofluids increase the convective heat transfer and thermal conductivity of the base fluids performance. In common liquids nanofluid is deferral of nano-particles. The classical conductivity theory of solid-fluid suspensions is used when the suspensions contain large-size particles. The theory cannot clarify that why nanoparticles of short concentrations can make better heat transfer of base liquids considerably bigger than the predication of model. Later Buongiorno [37] studied the convective heat transport in nanofluids in which he concluded that the Brownian and thermophoresis process will be imperative. Khanafer et al. [38] studied the buoyancy driven heat transfer effects in a channel with nanofluids. Letter on Buongiorno et al. [39] studied the experimental analysis of the nanofluid. Angayarkanni and Philip [40] presented a review on the thermal properties of nanofluid 
with some current developments. Several studies has been carried out for nanofluid inside parallel walls [41-52].

There are numerous agents who cause fluid movement in which some are more outstanding and conspicuous such as the pressure difference, the concentration difference, temperature difference, solid body movement and many more. In this work, the main focus on fluid movement, heat/mass transfer inside the peristaltic channel of deformable walls. The sources which will governs the fluid movement are the pressure gradient and movements of the rigid walls. The purpose of this study is to scrutinize the combined effects of the deformation and peristaltic movement of walls on fluid motion with heat/mass transfer effects in a channel. We have considered the nanofluid inside the channel walls, which are contracting/expanding in the normal direction and a sinusoidal wave is moving in the axial direction. The analytic solution of the non-linear problem have been presented by using perturbation technique. The outcomes are shown graphically and discussed physically. $\bar{h}(\bar{x}, \bar{t})=a(\bar{t})+b \sin \frac{2 \pi}{\lambda}(\bar{x}-c \bar{t})$,

in which $\lambda$ is wavelength and $b$ is amplitude. The governing equations are:

$\frac{\partial \bar{u}}{\partial \bar{x}}+\frac{\partial \bar{v}}{\partial \bar{y}}=0$,

$\frac{\partial \bar{u}}{\partial \bar{t}}+\bar{u} \frac{\partial \bar{u}}{\partial \bar{x}}+\bar{v} \frac{\partial \bar{u}}{\partial \bar{y}}=\frac{-1}{\rho} \frac{\partial \bar{p}}{\partial \bar{x}}+v\left(\frac{\partial^{2} \bar{u}}{\partial \bar{x}^{2}}+\frac{\partial^{2} \bar{u}}{\partial \bar{y}^{2}}\right)$

$\frac{\partial \bar{v}}{\partial \bar{t}}+\bar{u} \frac{\partial \bar{v}}{\partial \bar{x}}+\bar{v} \frac{\partial \bar{v}}{\partial \bar{y}}=\frac{-1}{\rho} \frac{\partial \bar{p}}{\partial \bar{y}}+v\left(\frac{\partial^{2} \bar{v}}{\partial \bar{x}^{2}}+\frac{\partial^{2} \bar{v}}{\partial \bar{y}^{2}}\right)$

$$
\begin{aligned}
& \frac{\partial \bar{T}}{\partial \bar{t}}+\bar{u} \frac{\partial \bar{T}}{\partial \bar{x}}+\bar{v} \frac{\partial \bar{T}}{\partial \bar{y}}=\frac{k}{\rho c_{p}}\left(\frac{\partial^{2} \bar{T}}{\partial \bar{x}^{2}}+\frac{\partial^{2} \bar{T}}{\partial \bar{y}^{2}}\right) \\
& \quad+\tau D_{B}\left(\frac{\partial \bar{T}}{\partial \bar{x}} \frac{\partial \bar{C}}{\partial \bar{x}}+\frac{\partial \bar{T}}{\partial \bar{y}} \frac{\partial \bar{C}}{\partial \bar{y}}\right)+\frac{\tau D_{T}}{T_{\infty}}\left(\left(\frac{\partial \bar{T}}{\partial \bar{x}}\right)^{2}+\left(\frac{\partial \bar{T}}{\partial \bar{y}}\right)^{2}\right)
\end{aligned}
$$

\section{Modelling}

Dynamics of nanofluid in semi-infinite channel having width $2 a$ is considered. One end is closed by nonporous elastic sheet, which shrinks/expands as the walls are deformed. The heated walls are expanding/contracting in the normal direction and sinusoidal wave of speed $c$ is generating on the surface of walls in the longitudinal direction, so the width between walls is function of time. The concentration and temperature on the lower and the upper plates are $T_{0}, C_{0}$ and $T_{1}, C_{1}$. The physical model is presented in Fig. 1.

Mathematically, the wall geometry is described as:

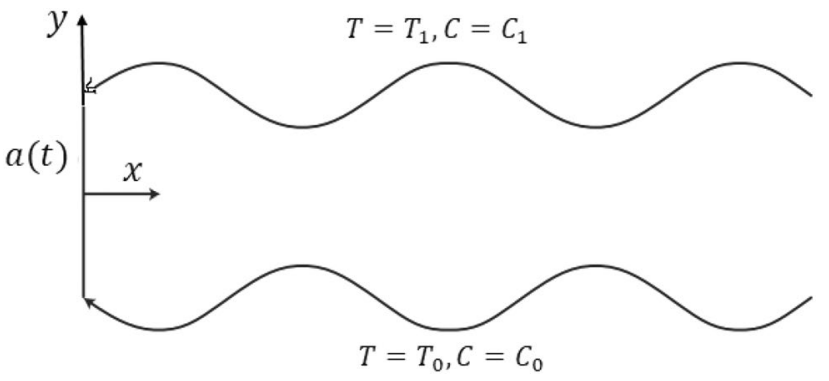

in which $(\bar{u}, \bar{v})$ represents velocities in the axial and normal directions $(\bar{x}, \bar{y}), \bar{p}$ is pressure, $\rho$ is density, $\bar{T}$ is temperature of the fluid, $\bar{C}$ is concentration, $D_{T}$ is thermophoresis diffusion coefficient and $D_{B}$ is Brownian diffusion coefficient. In order to transform the equations from laboratory frame into wave frame, we define the following new variables:

$\hat{x}=\bar{x}-c \bar{t}, \quad \hat{y}=\bar{y}, \quad \hat{u}=\bar{u}-c, \quad \hat{v}=\bar{v}, \quad \hat{p}=\bar{p}, \quad \hat{T}=\bar{T}, \quad \hat{C}=\bar{C}$.

Using the transformations (7) into Eqs. (2)-(6), we get

$\frac{\partial \hat{u}}{\partial \hat{x}}+\frac{\partial \hat{v}}{\partial \hat{y}}=0$,

$\frac{\partial \hat{u}}{\partial \hat{t}}+\hat{u} \frac{\partial \hat{u}}{\partial \hat{x}}+\hat{v} \frac{\partial \hat{u}}{\partial \hat{y}}=\frac{-1}{\rho} \frac{\partial \hat{p}}{\partial \hat{x}}+v\left(\frac{\partial^{2} \hat{u}}{\partial \hat{x}^{2}}+\frac{\partial^{2} \hat{u}}{\partial \hat{y}^{2}}\right)$,

$\frac{\partial \hat{v}}{\partial \hat{t}}+\hat{u} \frac{\partial \hat{v}}{\partial \hat{x}}+\hat{v} \frac{\partial \hat{v}}{\partial \hat{y}}=\frac{-1}{\rho} \frac{\partial \hat{p}}{\partial \hat{y}}+v\left(\frac{\partial^{2} \hat{v}}{\partial \hat{x}^{2}}+\frac{\partial^{2} \hat{v}}{\partial \hat{y}^{2}}\right)$

$$
\begin{aligned}
& \frac{\partial \hat{T}}{\partial \hat{t}}+\hat{u} \frac{\partial \hat{T}}{\partial \hat{x}}+\hat{v} \frac{\partial \hat{T}}{\partial \hat{y}}=\frac{k}{\rho c_{p}}\left(\frac{\partial^{2} \hat{T}}{\partial \hat{x}^{2}}+\frac{\partial^{2} \hat{T}}{\partial \hat{y}^{2}}\right) \\
& \quad+\tau D_{B}\left(\frac{\partial \hat{T}}{\partial \hat{x}} \frac{\partial \hat{C}}{\partial \hat{x}}+\frac{\partial \hat{T}}{\partial \hat{y}} \frac{\partial \hat{C}}{\partial \hat{y}}\right)+\frac{\tau D_{T}}{T_{\infty}}\left(\left(\frac{\partial \hat{T}}{\partial \hat{x}}\right)^{2}+\left(\frac{\partial \hat{T}}{\partial \hat{y}}\right)^{2}\right)
\end{aligned}
$$

Fig. 1 Schematic problem 


$$
\begin{aligned}
& \frac{\partial \hat{C}}{\partial \hat{t}}+\hat{u} \frac{\partial \hat{C}}{\partial \hat{x}}+\hat{v} \frac{\partial \hat{C}}{\partial \hat{y}}=D_{B}\left(\frac{\partial^{2} \hat{C}}{\partial \hat{x}^{2}}+\frac{\partial^{2} \hat{C}}{\partial \hat{y}^{2}}\right) \\
& +D_{T}\left(\frac{\partial}{\partial \hat{x}}\left(\frac{1}{T_{\infty}} \frac{\partial \hat{C}}{\partial \hat{x}}\right)+\frac{\partial}{\partial \hat{y}}\left(\frac{1}{T_{\infty}} \frac{\partial \hat{C}}{\partial \hat{y}}\right)\right) .
\end{aligned}
$$

The transformations and non-dimensional variables are $x=\frac{2 \pi \hat{x}}{\lambda}, \quad y=\frac{\hat{y}}{a}, \quad u=\frac{\hat{u}}{c}, \quad v=\frac{\hat{v}}{c}, \quad h=\frac{\hat{h}}{c}$,

$\psi=\frac{\hat{\psi}}{a c}, \quad T=\frac{\hat{T}-T_{0}}{T_{1}-T_{0}}, \quad C=\frac{\hat{C}-C_{0}}{C_{1}-C_{0}}$,

$u=\frac{\partial \psi}{\partial y}, \quad v=-\frac{\partial \psi}{\partial x}$.

The pressure terms are eliminated from Eqs. (9) and (10) by cross differentiation and subtracting and using Eq. (13), we get the vorticity equation.

$$
\begin{aligned}
& \alpha \nabla^{2} \psi_{y y}-2 \delta^{2} \alpha \psi_{x x}-\frac{a^{2}}{v} \nabla^{2} \psi_{t}+\alpha y \nabla^{2} \psi_{y} \\
& \quad+\operatorname{Re} \delta\left[-\psi_{y} \nabla^{2} \psi_{x}+\psi_{x} \nabla^{2} \psi_{y}\right]=-\left(\delta^{2} \nabla^{2} \psi_{x x}+\nabla^{2} \psi_{y y}\right),
\end{aligned}
$$

in which $\nabla^{2}=\delta^{2} \frac{\partial^{2}}{\partial x^{2}}+\frac{\partial^{2}}{\partial y^{2}}$. Substituting Eq. (13), into the above system of equations, Eq. (8) is identically satisfied and Eqs. (9)-(12) become under the assumption that the small parameter $\alpha$ remains the constant and let the stream function $\psi$ varies with $\alpha$ instead of $t$ [8], we get:

$$
\begin{aligned}
& \alpha \nabla^{2} \psi_{y y}-2 \delta^{2} \alpha \psi_{x x}+\alpha y \nabla^{2} \psi_{y} \\
& \quad+\operatorname{Re} \delta\left[-\psi_{y} \nabla^{2} \psi_{x}+\psi_{x} \nabla^{2} \psi_{y}\right]=-\left(\delta^{2} \nabla^{2} \psi_{x x}+\nabla^{2} \psi_{y y}\right),
\end{aligned}
$$

$-\alpha y \psi_{y y}+\operatorname{Re} \delta\left[\psi_{y} \psi_{y x}-\psi_{x} \psi_{y y}\right]=-\frac{\partial p}{\partial x}+\left(\delta^{2} \psi_{y x x}+\psi_{y y y}\right)$,

$-\alpha \psi_{x}+\alpha y \psi_{x y}+\operatorname{Re} \delta\left[-\psi_{y} \psi_{x x}+\psi_{x} \psi_{x y}\right]=-\frac{1}{\delta^{2}} \frac{\partial p}{\partial y}-\left(\delta^{2} \psi_{x x x}+\psi_{x y y}\right)$,

$$
\begin{aligned}
\psi= & \beta_{1} y-\beta_{2} y^{3}+\frac{y}{140 h}\left(210 F_{1}+14 \alpha h^{5} \beta_{2}+7 \alpha \delta \operatorname{Reh}^{5} \beta_{1}^{\prime} \beta_{2}-7 \alpha \delta \operatorname{Reh}^{5} \beta_{1} \beta_{2}^{\prime}+4 \delta \operatorname{Reh}^{7} \beta_{2}^{\prime} \beta_{2}\right) \\
& -\frac{y^{3}}{70 h^{3}}\left(35 F_{1}+14 \alpha h^{5} \beta_{2}+7 \delta \operatorname{Re}^{5} \beta_{1}^{\prime} \beta_{2}-7 \alpha \delta \operatorname{Re} h^{5} \beta_{1} \beta_{2}^{\prime}-7 \delta \operatorname{Reh}^{5} \beta_{2}^{\prime}+3 \delta \operatorname{Re} h^{7} \beta_{2}^{\prime} \beta_{2}\right) \\
& +\frac{y^{5}}{20}\left(2 \alpha \beta_{2}+\delta \operatorname{Re} \beta_{1}^{\prime} \beta_{2}-\delta \operatorname{Re} \beta_{1} \beta_{2}^{\prime}-\delta \operatorname{Re} \beta_{2}^{\prime}\right)+\frac{y^{7}}{70} \delta \operatorname{Re} \beta_{2}^{\prime} \beta_{2} .
\end{aligned}
$$

with boundary conditions:

In above boundary condition

$Q=F+1$, laboratory frame and

$F=\int_{0}^{h} \frac{\partial \psi}{\partial y} d y=\psi(h)-\psi(0)$, defined as:

$\psi(0)=0, \quad \psi_{y y}(0)=0, \quad \theta(0)=0, \quad \phi(0)=0$,

$\psi(h)=F, \quad \psi_{y}(h)=0, \quad \theta(h)=1, \quad \phi(h)=1$.

where $Q$ is the non-dimensional mean flow rate in the

where $h=1+\varphi \sin x$. Note that $\alpha$ is expansion/contraction parameter, the Reynolds number Re represents suction/ injection, the Prandtl number $\operatorname{Pr}$, the wave number $\delta$, the Brownian and the thermophrosis parameter $\mathrm{Nb}$ and $\mathrm{Nt}$ are

$$
\begin{aligned}
\alpha & =\frac{a \dot{a}}{v}, \quad \operatorname{Re}=\frac{c a_{0}}{v}, \quad \operatorname{Pr}=\frac{\mu c_{p}}{k}, \quad \delta=\frac{2 \pi a_{0}}{\lambda}, \\
N b & =\frac{\tau D_{B}\left(C_{1}-C_{0}\right)}{v}, \quad N t=\frac{\tau D_{T}\left(T_{1}-T_{0}\right)}{v T_{0}} .
\end{aligned}
$$

Equations (15)-(19) with conditions (20) can be solved by perturbation method and assumed that all the parameters are of the same order. Note that the perturbation solutions are valid for small value of all the governing parameters and they are accurate up to the order of $\alpha^{2}$ and $\delta^{2}$. Moreover, we get the following form for stream function, temperature and concentration: 


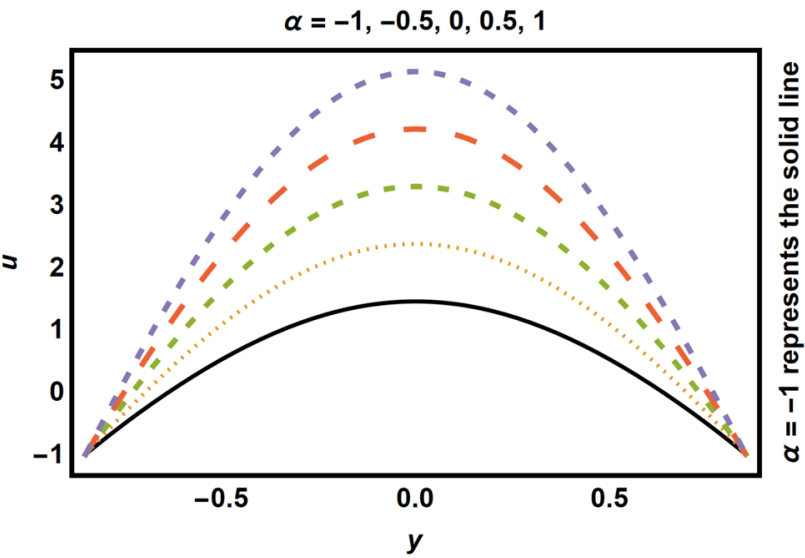

Fig. 2 The axial velocity $u(x, y)$ is graphed against $y$ for different and small values of wall expansion ratio

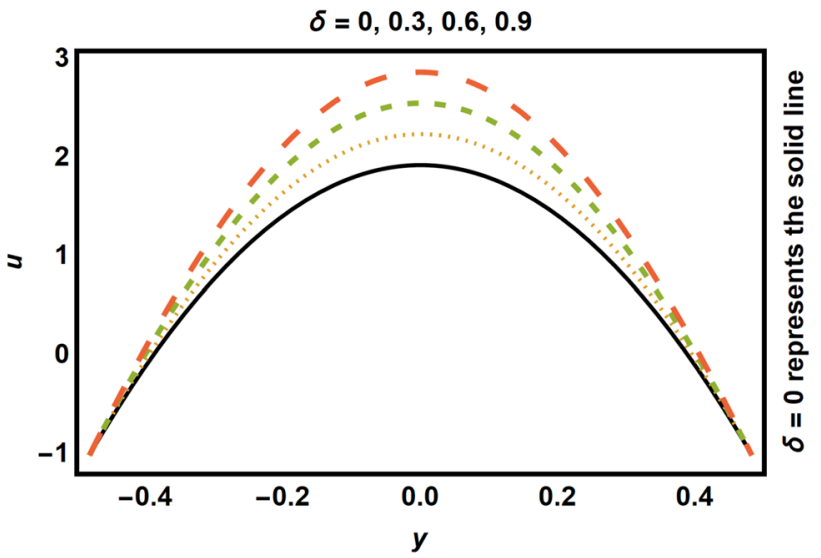

Fig. 3 The axial velocity $u(x, y)$ is graphed against $y$ for different and small values of wave number

$$
\begin{aligned}
\theta= & \beta_{7} y-\frac{\operatorname{Pr} y}{60}\left(10 \alpha h^{2} \beta_{7}+30 N \operatorname{th} \beta_{7}^{2}+30 N b h \beta_{7} \beta_{8}+10 \delta \operatorname{Reh}^{2} \beta_{1}^{\prime} \beta_{7}-3 \delta \operatorname{Reh}^{4} \beta_{2}^{\prime} \beta_{7}\right. \\
& \left.-\delta \operatorname{Re} h^{2}\left(10+10 \beta_{1}-9 h^{2} \beta_{2}\right) \beta_{7}^{\prime}\right)-\frac{\operatorname{Pr} y^{2}}{2}\left(N t \beta_{7}^{2}+N b \beta_{7} \beta_{8}\right) \\
& +\frac{\operatorname{Pr} y^{3}}{6}\left(\alpha \beta_{7}-\delta \operatorname{Re} \beta_{1}^{\prime} \beta_{7}+\delta \operatorname{Re} \beta_{7}^{\prime}\right)+\frac{\operatorname{Pr} y^{5}}{20} \delta \operatorname{Re}\left(\beta_{2}^{\prime} \beta_{7}-3 \beta_{7}^{\prime} \beta_{2}\right) .
\end{aligned}
$$

$$
\begin{aligned}
\phi= & \beta_{8} y+\frac{\operatorname{Leh}}{60}\left(10 \frac{N t}{N b} \alpha h \operatorname{Pr} \beta_{7} \beta_{8}-10 \alpha h \beta_{8}+30 \frac{N t^{2}}{N b} \operatorname{Pr} \beta_{7}^{2}+3 N t \operatorname{Pr} \beta_{7} \beta_{8}\right. \\
& \left.+\delta \operatorname{Re} h^{2}\left(10 \frac{N t}{N b} \operatorname{Pr} \beta_{1}^{\prime} \beta_{7}-10 \beta_{1}^{\prime} \beta_{8}-3 \frac{N t}{N b} h^{2} \operatorname{Pr} \beta_{2}^{\prime} \beta_{7}+3 h^{2} \beta_{2}^{\prime} \beta_{8}\right)-\left(10+10 \beta_{1}-9 h^{2} \beta_{2}\right)\left(\frac{N t}{N b} \operatorname{Pr} \beta_{7}^{\prime}-\beta_{8}^{\prime}\right)\right) \\
& +\frac{N t}{2 N b} \operatorname{Le} \operatorname{Pr} \beta_{7} y^{2}\left(N t \beta_{7}+N b \beta_{8}\right)-\frac{\operatorname{Ley}^{3}}{6}\left(\alpha \frac{N t}{N b} \operatorname{Pr} \beta_{7}-\alpha \beta_{8}+\delta \frac{N t}{N b} \operatorname{Re} \beta_{1} \beta_{7}-\delta \operatorname{Re} \beta_{1}^{\prime} \beta_{8}-\delta \frac{N t}{N b} \operatorname{Pr} \operatorname{Re} \beta_{7}^{\prime}\right. \\
& \left.-\delta \frac{N t}{N b} \operatorname{Pr} \operatorname{Re} \beta_{1} \beta_{7}^{\prime}+\delta \operatorname{Re} \beta_{8}^{\prime}+\delta \operatorname{Re} \beta_{1} \beta_{8}^{\prime}\right)-\frac{\delta \operatorname{Le} \operatorname{Re} y^{5}}{20}\left(-\delta \frac{N t}{N b} \operatorname{Pr} \beta_{2}^{\prime} \beta_{7}-\beta_{2}^{\prime} \beta_{8}-\delta \frac{N t}{N b} \operatorname{Pr} \beta_{2} \beta_{7}^{\prime}+3 \beta_{2} \beta_{8}^{\prime}\right) .
\end{aligned}
$$

where $\beta_{1}=\frac{3 F_{0}+h}{2 h}, \beta_{2}=\frac{F_{0}+h}{2 h^{3}}, \beta_{7}=\frac{1}{h}$ and $\beta_{8}=\frac{1}{h}$.

Note that the solution in Eq. (26) is exactly matched with the published results of Khan Marwat and Asghar [35] whereas the modeled problem presents a viscous flow in a deformable channel of peristaltically moving walls. The current formulation enhances flow heat and mass transfer of nanofluids in such channels.

\section{Graphical results and discussion}

This section presents analysis of different quantities on the velocity, the temperature and the concentration profiles, pressure gradient and pressure rise per wavelength. The problem in Eqs. (23)-(26) is solved with the help of perturbation method and the series solution is found. The perturbation solutions are effective for small values of involved parameters in the problem. The existing solution are confirmed and validated by comparing it with the published results for specific values of the governing parameters. In Fig. 2, axial velocity profiles are plotted against the dimensionless variable $y$ for diverse values of wall expansion ratio $\alpha$. The velocity is increased with increasing of $\alpha$. The axial velocity profiles are parabolic in nature and symmetrical about the center line of the channel. The gape among different profiles is maximum at the center whereas it is almost zero near both walls. This fluid velocity is not zero at the walls because both the walls are moving with the velocity of wave speed $c$. Similarly in Fig. 3 effects of wave number $\delta$ are seen on the axial velocity and it is increased with increasing values of $\delta$. In Fig. 4, the effects of Reynolds number Re are shown on the axial velocity. 


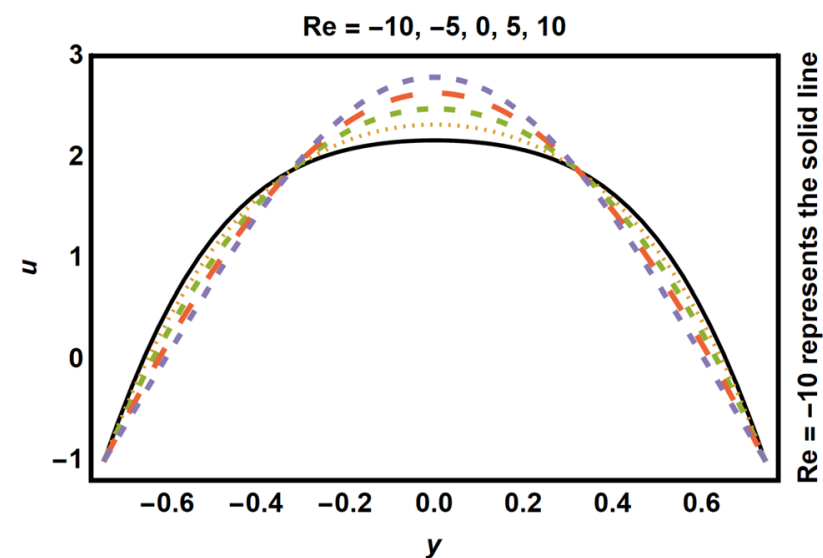

Fig. 4 The axial velocity $u(x, y)$ is graphed against $y$ for different values of suction injection parameter

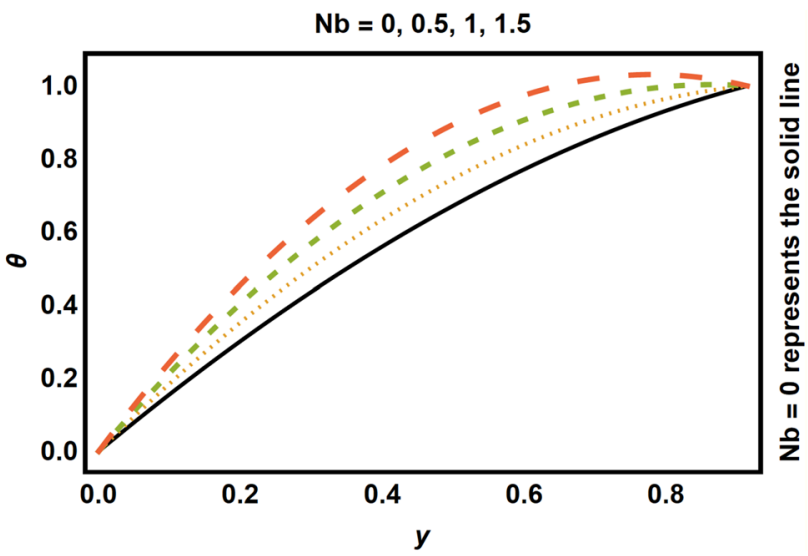

Fig. 5 The temperature distribution $\theta(x, y)$ is graphed against $y$ for diverse and small values of $\mathrm{Nb}$

$\mathrm{Nt}=0,0.5,1,1.5$

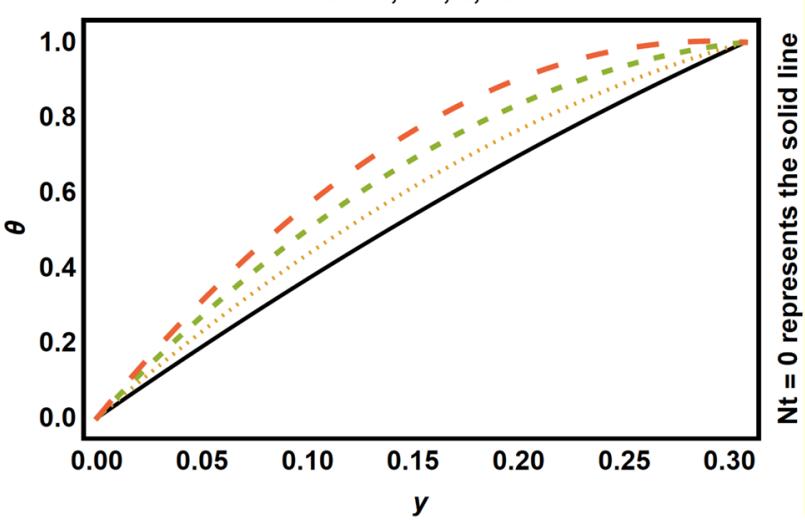

Fig. 6 The temperature distribution $\theta(x, y)$ is graphed against $y$ for diverse and small values of $\mathrm{Nt}$

\section{SN Applied Sciences}

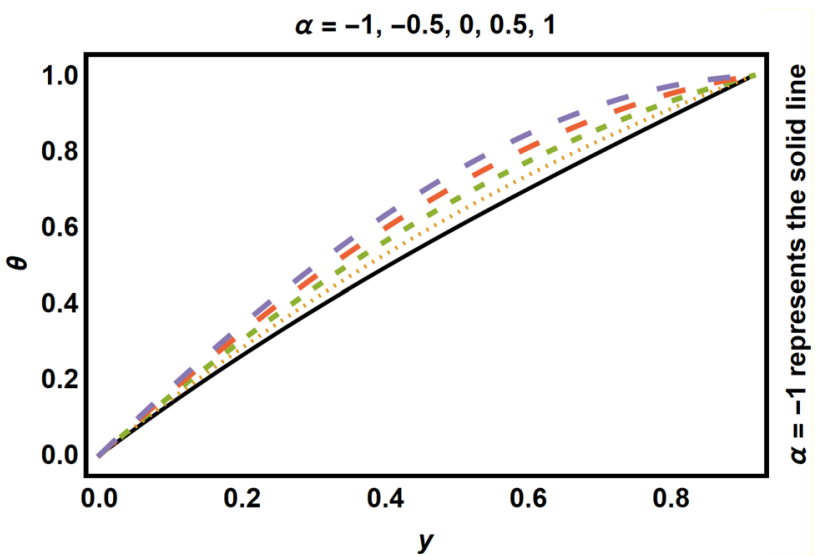

Fig. 7 The temperature distribution $\theta(x, y)$ is graphed against $y$ for different and small values of wall expansion ratio

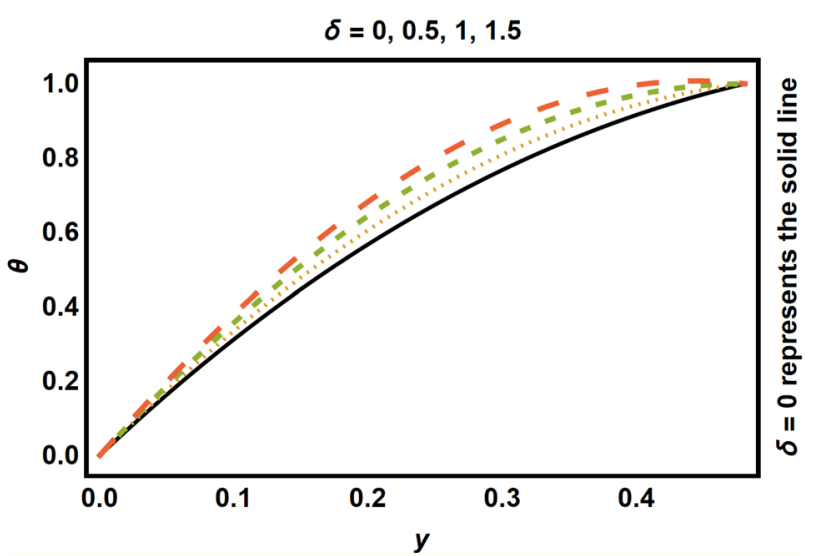

Fig. 8 The temperature distribution $\theta(x, y)$ is graphed against $y$ for diverse and small values of wave number

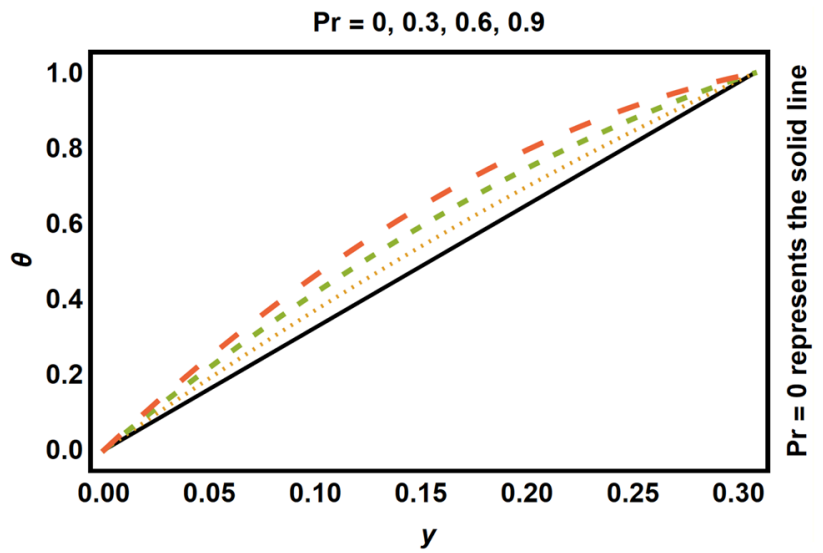

Fig. 9 The temperature distribution $\theta(x, y)$ is graphed against $y$ for diverse values of Prandtl number 


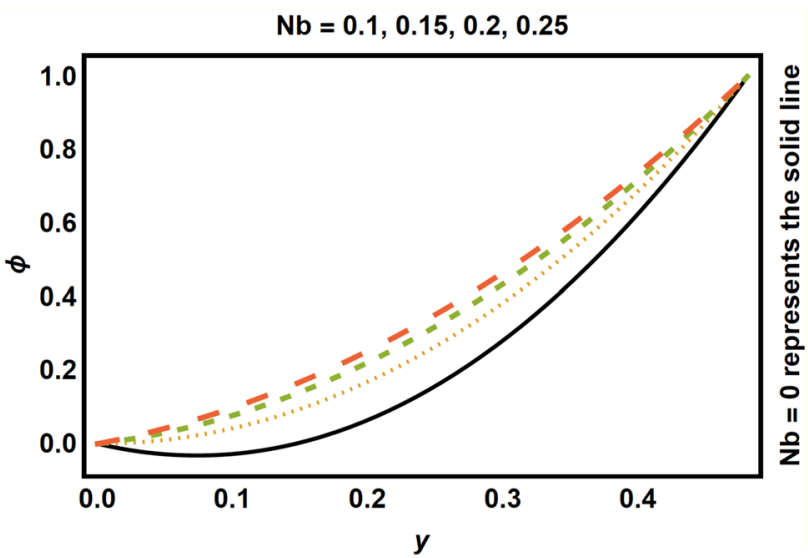

Fig. $10 \phi(x, y)$ is graphed against $y$ for diverse and small values of $\mathrm{Nb}$

$\mathrm{Nt}=0,0.5,1,1.5$

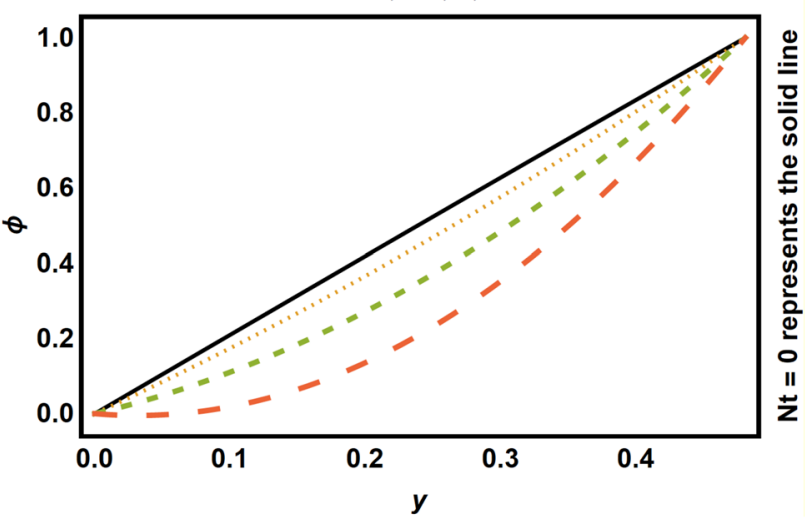

Fig. $11 \phi(x, y)$ is graphed against $y$ for diverse and small values of $N t$

For the case of suction, i.e. for negative values of Reynolds number, the velocity near the boundaries decreases while it surges in the center of the channel.

In Figs. 5, 6, 7, 8 and 9, the effects of $N b, N t, \alpha, \delta$ and $\operatorname{Pr}$ are seen on temperature distribution. Note the heat flow is discussed in the upper half of the channel. Figure 5 shows effects of $\mathrm{Nb}$ are observed on the profiles of temperature. For $\mathrm{Nb}=0$, the profile is exactly linear and temperature is increased with the increasing of $\mathrm{Nb}$. The profiles for $\mathrm{Nb}>0$ are of curved shape and changed uniformly. This variation in temperature is actually caused by Brownian motion of molecules and it is the arbitrary motion of particles suspended in the fluid which results from their collision with the fast-moving molecules in the fluid. The more energetic is the Brownian motion of molecules, the higher the temperature that we sense. More specifically, absolute temperature is relative to kinetic energy of the Brownian motion of molecules per unit mass. If we raise the temperature, Brownian motion becomes more energetic. On the other

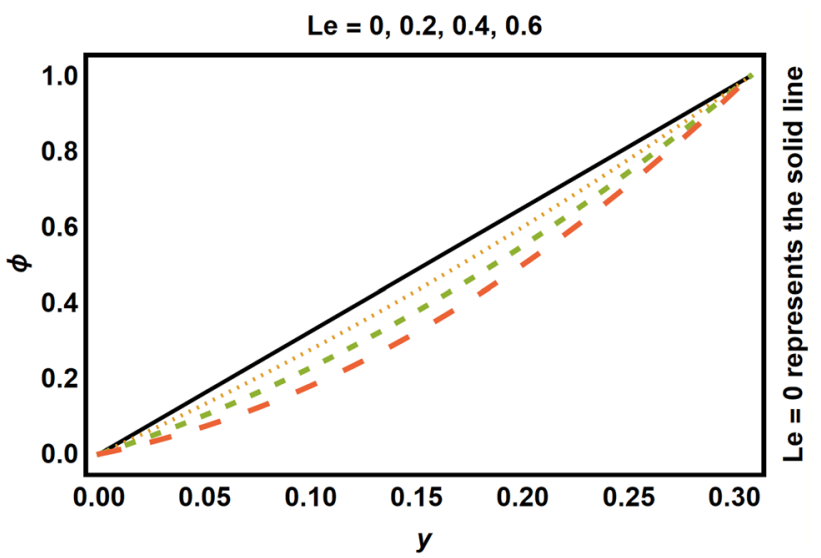

Fig. 12 The concentration distribution $\phi(x, y)$ is graphed against $y$ for diverse and small values of Le

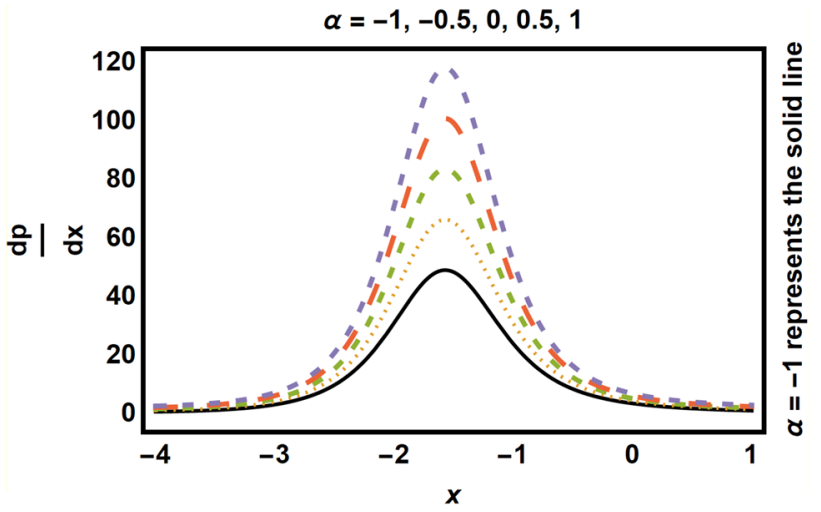

Fig. 13 The axial pressure gradient $d p / d x$ is graphed against $x$ for different and small values of wall expansion ratio

hand, if Brownian motion is increasing then temperature will be increased. This confirms the direct relation between Brownian motion and temperature. Thermophoresis is the effective source produced by temperature difference between the hot gas and the cold surfaces. It also controls the particulate movement towards the cold wall. It is noted that the temperature distribution is changed with the deviation of thermophoresis parameter. The graphs in Fig. 6 shows that temperature is increased with the growing of thermophoresis parameter $N t$. For $N t=0$, the temperature profiles are closed to linear and direct relation between Thermophoresis parameter $\mathrm{Nt}$ and temperature profiles is also confirmed. The effects of wall expansion ratio $\alpha$ are also studied on the temperature profiles in Fig. 7. It is decreased with the increasing of wall expansion ratio. The only possible reason for decreasing in temperature is the increasing gape between walls. The effects of wave number $\delta$ are shown on the temperature profiles in Fig. 8. If wave number increases then temperature will be decreased. This means that temperature and wave number 


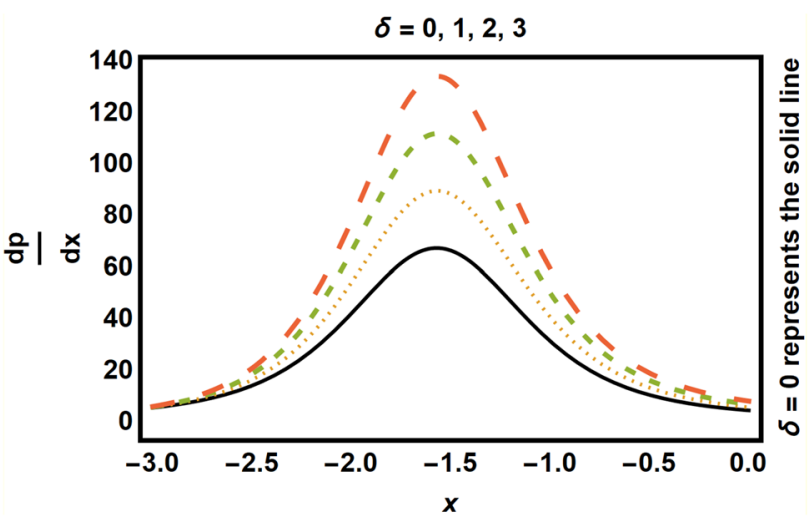

Fig. 14 The axial pressure gradient $d p / d x$ is graphed against $x$ for different and small values of wave number

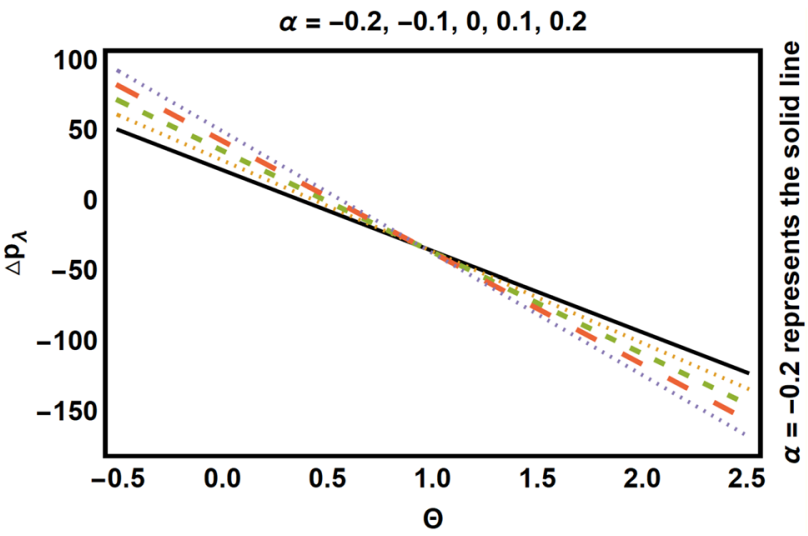

Fig. 15 The pressure rise per wavelength $\Delta p_{\lambda}$ is graphed against $\Theta$ for diverse and small values of $\alpha$

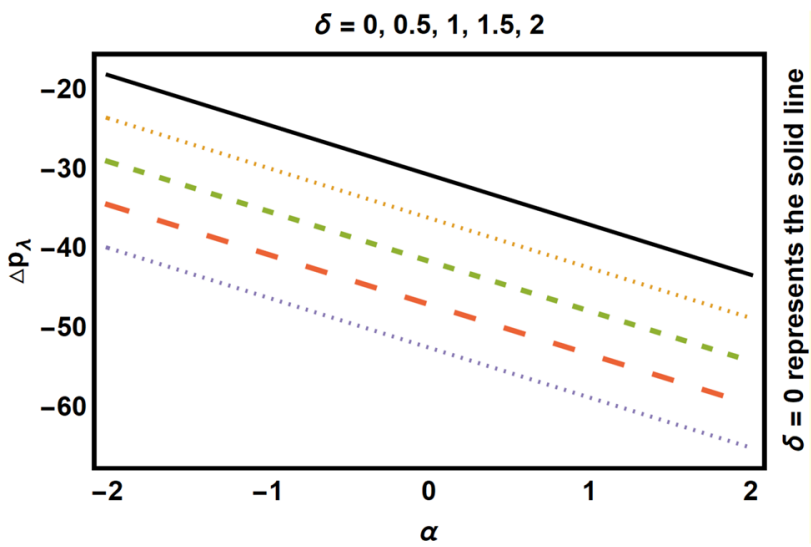

Fig. 16 The pressure rise per wavelength $\Delta p_{\lambda}$ is graphed against $\Theta$ for diverse values of $\delta$

are inversely proportional. It is noted that the temperature is more sensitive to $N b, \operatorname{Pr}$ and $N t$ whereas the variation in its graphs with $\delta$ and $\alpha$ is pronounced near the upper wall.
In Fig. 9 the effects of Prandtl number are shown on the temperature distribution.

In Figs. 10, 11 and 12, the effects of $N b, N t$ and Le are seen on concentration distribution. It is noted that by growing the Brownian motion $\mathrm{Nb}$, increases the concentration profile whereas, the increasing Thermophoresis parameter $\mathrm{Nt}$, decreases the concentration profile. Thus greater concentration of nanoparticles corresponds to higher $\mathrm{Nb}$, and lower thermophrosis diffusion $\mathrm{Nt}$ which can be seen from Figs. 10 and 11 respectively. The influence of Le on the concentration profiles are shown in Fig. 12 in the presence of wall expansion ration. It is clear that the concentration decreased with the increasing of Le. For $L e=0$, the concentration profiles are linear.

In the next two figures, effects of wall deformation ratio $\alpha$ and wave number $\delta$ are seen on axial pressure gradient. In Fig. 13, the axial pressure gradient is graphed against the axial distance for different values of wall deformation ratio. It is changed sinusoidally and increased with the increasing of $\alpha$. The amplitude of the sinusoidal pressure (axial) gradient is rapidly changed with the increasing values of wall expansion ratio. However, it is decreased for increasing the wall contraction ratio. Similarly, the same pressure gradient is also plotted in Fig. 14 against the axial distance for different values of wave number and once again the sinusoidal behavior of the pressure gradient is observed. It is increased instantly with the increase of this parameter. For large values of $\delta$, the wave length and amplitude of each profile is greater than the other, which correspond to the smaller values. In Fig. 15 pressure rise per wavelength is evaluated and graphed against volume flow rate $\Theta$ for different and small values of the wall deformation rate. The pressure drop against $\Theta$ is linear for each value of wall deformation rate. It is increased/decreased for increasing the wall expansion ratio $\alpha>0$ /wall contraction ratio $\alpha<0$. In Fig. 16 pressure rise per wavelength is evaluated and graphed against volume flow rate $\Theta$ for different and small values of wave number. The pressure drop against $\Theta$ is linear for each value of the wave number. The profiles in this figure are exactly matched with the classical profiles published in [35].

\section{Concluding remarks}

Peristaltic flow with double diffusion of a nanofluid in a deformable channel of heated porous walls is investigated in this paper. Perturbation method provides a satisfactory solution for the field quantities in terms of the characterizing parameters. The variation of these parameters describes the flow heat and mass transfer behavior in a deformable channel of heated and porous walls. The flow profiles and pressure gradient are calculated and effects of 
the additional characterizing parameters are thoroughly investigated. These parameters in combination with the deforming parameter provide a useful analytical understanding of the flow heat and mass transfer behavior in deforming channels. The insight provided by the study will be useful both in applications of the deforming channels and tubes when the additional considerations of heat and mass transfer phenomena are incorporated besides the mathematical value of the analytical solution. A detailed impact of the characterizing parameters has been narrated in "Results and Discussion".

\section{Compliance with ethical standards}

Conflict of interest The authors declare that they have no conflict of interest.

\section{References}

1. Berman AS (1953) Laminar flow in channels with porous walls. J Appl Phys 24:1232-1235

2. Sellars JR (1955) Laminar flow in channels with porous walls at high suction Reynolds numbers. J Appl Phys 26:489-490

3. Yuan S (1956) Further investigation of laminar flow in channels with porous walls. J Appl Phys 27:267-269

4. Proudman I (1960) An example of steady laminar flow at large Reynolds number. J Fluid Mech 9:593-602

5. Terrill RM (1965) Laminar flow in a uniformly porous channel with large injection. Aeronaut Q 16:323

6. Cox SM (1991) Two-dimensional flow of a viscous fluid in a channel with porous walls. J Fluid Mech 227:1-33

7. Uchida S, Aoki H (1977) Unsteady flows in a semi-infinite contracting or expanding pipe. J Fluid Mech 82:371-387

8. Goto M, Uchida S (1990) Unsteady flows in a semi-infinite contracting or expanding pipe with injection through wall. Trans Jpn Soc Aeronaut Space Sci 33:14-27

9. Dauenhauer EC, Majdalani J (1999) Unsteady flows in semiinfinite expanding channels with wall injection. In: AIAA 99, p 3523

10. Dauenhauer EC, Majdalani J (2001) Exact self-similarity solution of the Navier-Stokes equations for a deformable channel with wall suction or injection. AIAA 2001, p 3588

11. Majdalani J, Zhou C, Dawson CA (2002) Two-dimensional viscous flow between slowly expanding or contracting walls with weak permeability. J Biomech 35:1399-1403

12. Majdalani J, Zhou C (2003) Moderate-to-large injection and suction driven channel flows with expanding or contracting walls. Z Angew Math Phys 83:181-196

13. Boutros YZ, Abd-el-Malek AB, Badran NA, Hassan HS (2007) Lie group method solution for two-dimensional viscous flow between slowly expanding or contracting walls with weak permeability. Appl Math Model 31:1092-1108

14. Asghar S, Mushtaq M, Kara AH (2008) Exact solutions using symmetry methods and conservation laws for the viscous flow through expanding-contracting channels. Appl Math Model 32:2936-2940

15. Mahmood M, Hossain MA, Asghar S, Hayat T (2008) Application of Homotopy perturbation method to deformable channel with wall suction and injection in a porous medium. Int J Nonlinear Sci Numer Simul 9:195-206

16. Matebese BT, Adem AR, Khalique CM, Hayat T (2010) Two-dimensional flow in a deformable channel with porous medium and variable magnetic field. Math Comput Appl 15:674-684

17. Si X, Zheng L, Zhang X, Chao Y (2010) Perturbation solution to unsteady flow in a porous channel with expanding or contracting walls of a transverse magnetic field. Appl Math Mech 31:151-158

18. Si XH, Zheng LC, Zhang XX, Li M, Yang JH, Chao Y (2011) Multiple solutions for the laminar flow in a porous pipe with suction at slowly expanding or contracting wall. Appl Math Comput 28:3515-3521

19. Srinivas S, Reddy AS, Ramamohan TR (2012) A study on thermaldiffusion and diffusion-thermo effects in a two-dimensional viscous flow between slowly expanding or contracting walls with weak permeability. Int J Heat Mass Transf 55:3008-3020

20. Srinivas S, Reddy AS, Ramamohan TR, Shukla AK (2014) Influence of heat transfer on MHD flow in a pipe with expanding or contracting permeable wall. Ain Shams Eng J 5:817-830

21. Jafaryar M, Farkhadina F, Mohammadian E, Hosseini M, Khazaee AM (2014) Analytical investigation of laminar flow through expanding or contracting gaps with porous walls. Propul Power Res 3:222-229

22. Asghar S, Abbas Z, Mushtaqm M, Hayat T (2016) Flow and heat transfer analysis in a deformable channel. J Eng Phys Thermop 89:929-941

23. Latham TW (1966) Fluid motion in a peristaltic pump. MS thesis, MIT, Cambridge

24. Burns JC, Pareks T (1967) Peristaltic motion. J Fluid Mech 29:731-743

25. Fung YC, Yih CS (1968) Peristaltic transport. ASME J Appl Mech 35:669-675

26. Yin F, Fung YC (1969) Peristaltic waves in circular tubes. ASME J Appl Mech 36:579-587

27. Shapiro AH, Jaffrin MY, Weinberg SL (1969) Peristaltic pumping with long wavelengths at low Reynolds number. J Fluid Mech 37:799-825

28. Weinberg SL, Eckstein EC, Shapiro AH (1971) An experimental study of peristaltic pumping. J Fluid Mech 49:461-479

29. Yin FCP, Fung YC (1971) Comparison of theory and experiment in peristaltic transport. J Fluid Mech 47:93-112

30. Jaffrin MY, Shapiro AH (1971) Peristaltic pumping. Annu Rev Fluid Mech 3:13-37

31. Takabatake S, Ayukawa K (1982) Numerical study of two-dimensional peristaltic flows. J Fluid Mech 122:439-465

32. Pozrikidis C (1987) A study of peristaltic flow. J Fluid Mech 180:515-527

33. Mekheimer KS (2003) Non-linear peristaltic transport of magnetohydrodynamic flow of an inclined planar channel. Arab J Sci Eng 28:183-201

34. Mishra M, Rao AR (2003) Peristaltic transport of a Newtonian fluid in an asymmetric channel. Z Angew Math Phys 54:532-550

35. Khan Marwat DN, Asghar S (2011) Peristaltic flow in a deformable channel. Z Naturforsch A 66:24-32

36. Choi SUS (1995) Enhancing thermal conductivity of fluids with nanoparticles. In: Wang DA (ed) Developments and applications of non-Newtonian flows, FED-231/MD-66. ASME, New York, pp 99-105

37. Buongiorno J (2010) Convective transport in nanofluids. ASME J Heat Transf 128:240-250

38. Khanafer K, Vafai K, Lightstone M (2003) Buoyancy-driven heat transfer enhancement in a two-dimensional enclosure utilizing nanofluids. Int J Heat Mass Transf 46:3639-3653

39. Buongiorno J, Venerus DC, Prabhat N, McKrell T, Townsend J, Christianson R, Tolmachev YV, Keblinski P, Hu LW, Alvarado JL, 
Bang IC, Bishnoi SW, Bonetti M, Botz F, Cecere A, Chang Y, Chen G, Chen H, Chung SJ, Chyu MK, Das SK, Paola RD, Ding Y, Dubois F, Dzido G, Eapen J, Escher W, Funfschilling D, Galand Q, Gao J, Gharagozloo PE, Goodson KE, Gutierrez JG, Hong H, Horton M, Hwang KS, lorio CS, Jang SP, Jarzebski AB, Jiang Y, Jin L, Kabelac S, Kamath A, Kedzierski MA, Kieng LG, Kim C, Kim JH, Kim S, Lee SH, Leong KC, Manna I, Michel B, Ni R, Patel HE, Philip J, Poulikakos D, Reynaud C, Savino R, Singh PK, Song P, Sundararaian T, Timofeeva E, Tritcak T, Turanov AN, Vaerenbergh SV, Wen D, Witharana S, Yang C, Yeh WH, Zhao XZ, Zhou SQ (2009) A benchmark study on the thermal conductivity of nanofluids. J Appl Phys 106:094312-14

40. Angayarkanni SA, Philip J (2015) Review on thermal properties of nanofluid: recent developments. Adv Colloid Interface Sci 225:146-176

41. Srinivas S, Vijayalakshmi A, Reddy AS, Ramamohan TR (2016) MHD flow of a nanofluid in an expanding or contracting porous pipe with chemical reaction and heat source/sink. Propul Power Res 5:134-148

42. Ahmed N, Jhan U, Din STM (2017) MHD nanofluid flow through a deformable asymmetric porous channel. Eng Comput 34:852-868

43. Fakour M, Ganji DD, Khalili A, Bakhshi A (2017) Heat transfer in nanofluid MHD flow in a channel with permeable walls. Heat Transf Res 48:221-238

44. Hayat T, Sajjad R, Alsaedi A, Muhammad T, Ellahi R (2017) On squeezed flow of couple stress nanofluid between two parallel plates. Res Phys 7:553-561

45. Sobamowo MG, Akinshilo AT (2018) On the analysis of squeezing flow of nanofluid between two parallel plates under the influence of magnetic field. Alex Eng J 57:1413-1423
46. Animasaun IL, Mahanthesh B, Jagun AO, Bankole TD, Sivaraj $R$, Shah NA, Saleem S (2019) Significance of Lorentz force and thermoelectric on the flow of $29 \mathrm{~nm} \mathrm{CuO-water} \mathrm{nanofluid} \mathrm{on}$ an upper horizontal surface of a paraboloid of revolution. J Heat Transf 141:022402

47. Ali A, Shehzadi K, Sulaiman M, Asghar S (2019) Heat and mass transfer analysis of 3D Maxwell nanofluid over an exponentially stretching surface. Phys Scr 94:065206

48. Saleem S, Nadeem S, Rashidi MM, Raju CSK (2019) An optimal analysis of radiated nanomaterial flow with viscous dissipation and heat source. Microsyst Technol 25:683-689

49. Saleem S, Firdous H, Nadeem S, Khan AU (2019) Convective heat and mass transfer in magneto Walter's B nanofluid flow induced by a rotating cone. Arab J Sci Eng 44:1515-1523

50. Ali A, Sajjad A, Asghar S (2019) Thermal-diffusion and diffusionthermo effects in a nanofluid flow with non-uniform heat flux and convective walls. J Nanofluids 8:1367-1372

51. Sadiq MA, Khan AU, Saleem S, Nadeem S (2019) Numerical simulation of oscillatory oblique stagnation point flow of a magneto micropolar nanofluid. RSC Adv 9:4751-4764

52. Shahzadi I, Suleman S, Saleem S, Nadeem S (2020) Utilization of Cu-nanoparticles as medication agent to reduce atherosclerotic lesions of a bifurcated artery having compliant walls. Comput Methods Programs Biomed 184:105123

Publisher's Note Springer Nature remains neutral with regard to jurisdictional claims in published maps and institutional affiliations. 